\title{
Identidad regional y modelo de desarrollo alternativo: la Patagonia verde de Aysén*
}

\section{Regional Identity and Alternative Development Model: Aysén's Green Patagonia}

\author{
Carlos Fabián Pressacco *** \\ Esteban Valenzuela ${ }^{* * *}$ \\ Gonzalo Soto ${ }^{* * * *}$
}

Recibido: 27 de octubre de 2016

Aprobado: 14 de diciembre de 2016

Disponible en línea: 30 de enero de 2017

\section{Resumen}

Chile experimenta, desde hace varias décadas, un proceso de descentralización que ha fortalecido la autonomía de las regiones. Se trata, no obstante, de un proceso gradual que no ha logrado modificar de manera significativa el carácter centralista de su modelo estatal. Si bien dicho proceso cuenta con un marco general decidido en el Estado central, se expresa de manera específica de acuerdo con las especificidades de cada territorio. En tal sentido, el artículo explora el proceso de fortalecimiento

\begin{abstract}
Chile experiences, from several decades ago, a decentralizing process that has strengthened its regions' autonomy. However, this is a gradual process that has not substantially modified the centralist character of its state model. While this process has a general framework decided at the central state, it develops specifically according to the particularities of each territory. In this sense, the article explores the process of regional strengthening of the Aysén region in Chile, with three main aims: a) to
\end{abstract}

doi:10.11144/Javeriana.papo22-1.irym

* Artículo de revisión

** Licenciado en Ciencia Política por la Universidad Católica de Córdoba, especialista en Análisis y Administración de Políticas Estatales por la misma universidad, magíster en Ciencias Sociales por la Universidad Alberto Hurtado de Chile y doctor en Ciencias Políticas y Sociología por la Universidad de Deusto. Académico del Departamento de Ciencia Política y Relaciones Internacionales de la Universidad Alberto Hurtado. Correo electrónico: fpressac@ uahurtado.cl Orcid: http://orcid.org/0000-0001-9958-5376

*** Periodista por la Pontificia Universidad Católica de Chile, magíster en Ciencia Política por la misma universidad y doctor en Historia Contemporánea por la Universitat de València. Académico del Departamento de Ciencia Política y Relaciones Internacionales de la Universidad Alberto Hurtado. Correo electrónico: evalenzu@uahurtado. cl Orcid: http://orcid.org/0000-0003-1755-3309

**** Licenciado en Ciencia Política y Relaciones Internacionales por la Universidad Alberto Hurtado. Correo electrónico: gonzalo.soto@gmail.com Orcid: http://orcid.org/0000-0002-5238-7935 
regional en una de las regiones del Chile, Aysén, con tres objetivos principales: 1) indagar sobre el proceso de construcción de identidad regional asociado a la idea de Patagonia, 2) vincular ese proceso identitario con la elaboración de un modelo de desarrollo sustentable y 3 ) analizar el proceso de politización y la conformación de una coalición social-empresarial local que se opuso a megarrepresas hidroeléctricas y que contó con apoyo de redes internacionales. En cuanto a los resultados, observamos que la región de Aysén experimenta un proceso de politización sobre la base de la constitución de una identidad socioterritorial que se diferencia en dos aspectos centrales: 1) la apropiación de "lo patagónico" como sinónimo de "intocado" y 2) el intento por sentar las bases de un modelo de desarrollo alternativo. Una de las conclusiones principales del artículo es que la región de Aysén es la más permeable a la búsqueda de cooperación entre las regiones chilenas y argentina que configuran dicho territorio con una creciente identidad ecoturística a escala planetaria.

\section{Palabras clave:}

Patagonia; desarrollo alternativo; Aysén; identidad regional

\section{Cómo citar este artículo:}

Pressacco, C. F., Valenzuela, E. y Soto, G. (2017). Identidad regional y modelo de desarrollo alternativo: la Patagonia verde de Aysén. Papel Político, 22(1), 159-182. https://doi. org/10.11144/Javeriana.papo22-1.rrym inquire into the process of identity construction associated to the idea of Patagonia; b) to link such identity process with the definition of a model of sustainable development; and c) to analyze the politicization process and the configuration of a social-business coalition that opposed hydroelectric mega-dams and was supported by international networks. Regarding the research results, we observe that the Aysén region experiences a politization process on the basis of constituting a socio-territorial identity that is distinguished by two key aspects: the appropriation of the "patagonic" as a synonym of the "untouched" and the attempt to set the bases of an alternative development model. One of the main conclusions is the Aysén region is the most permeable to the pursuit of cooperation in the Chilean and Argentinian regions that configure this territory, with a growing ecotouristic identity on a global scale.

\section{Keywords:}

Patagonia; alternative development; Aysén; regional identity 


\section{Introducción}

El modelo de desarrollo imperante en Chile está crecientemente expuesto a un conjunto de tensiones de diversa naturaleza. Tras el término del modelo desarrollista, y en el régimen autoritario inaugurado en la década de 1970, el modelo de desarrollo dejó atrás el rol protagónico del Estado y el esfuerzo industrializador con una economía regulada y protegida orientada a asegurar las rentas de la burguesía nacional por medio de un consenso social y político que, a cambio de aquello, promovía mejoras sociales en una economía de carácter capitalista.

Aysén, al oponerse a la construcción de grandes centrales hidroeléctricas, se sumó a los movimientos que han cuestionado en Chile la matriz extractivista de las empresas mineras, eléctricas, pesqueras y forestales (Penaglia y Valenzuela, 2015). Aysén irrumpe como una sociedad que demanda otro modelo de desarrollo, alternativo al instalado por el autoritarismo neoliberal, caracterizado por una economía abierta primario-exportadora, desregulada, privatista. Esta región no quiere ser un territorio commodity de pesca y electricidad (Daher, 2003) afectado recurrentemente por los efectos nocivos de la industria del salmón (Ramos, 2014; Bustos, 2012). En Aysén, se desarrolla un conflicto en torno al modelo de desarrollo que debe prevalecer: o un territorio que debe explotar sus ventajas comparativas para la producción a gran escala de actividades extractivistas o una zona de resistencia que explora un viraje ambiental y un modelo ecoturístico con pacto social de redes microempresariales (Lander, 2014; Portillo, 2014).

La centralidad reconocida al mercado como espacio regulador del sistema social significó el debilitamiento del Estado - y de la política- como sujeto de coordinación, e implicó la supresión de un débil esquema de derechos sociales y la ampliación de los márgenes de ganancia de la empresa en un contexto agravado por el centralismo político chileno que ahondó disparidades regionales y el aumento de los liderazgos contenciosos para hacer oír su voz (Valenzuela, 2015).

Más allá de las especificidades propias de cada momento histórico, hay dos aspectos que evidencian elementos de continuidad: por un lado, tanto en el modelo industrializador como en el primario exportador, el descuido por la dimensión medioambiental; por otro, y más allá de los tibios avances en el fortalecimiento de los Gobiernos subnacionales, un esquema de toma de decisiones altamente centralizado desde una triple perspectiva: 1) Estado unitario, 2) presidencialismo y 3) sistema de partidos nacionalizado.

Si bien es posible identificar rastros de un malestar en gestación sobre el modelo de desarrollo, aquellos se hacen evidentes a partir de la gestación de un conjunto de movimientos sociales que cuestionan abiertamente este esquema. El movimiento estudiantil, los ecologistas y medioambientales, así como los territoriales, son portadores de una creciente conflictividad. Los movimientos de Aysén, Magallanes, Freirina, Calama, Caimanes y el más reciente de Chiloé dan cuenta del creciente descontento ciudadano centrado en 
dos elementos: 1) la imposibilidad de ser sujetos en la decisión sobre lo que sucede en el territorio y 2) los costos derivados de un modelo de desarrollo extractivista y depredador.

Este artículo explora tal proceso de creciente conflictividad con el foco puesto en el movimiento de Aysén y en el proceso de constitución de sujetos sociales y políticos territoriales y, específicamente, en el de la identidad que se gesta a partir de una particular y compartida visión sobre el significado del territorio. No se trata de una identidad definida y consolidada que deja atrás su anterior configuración. Más bien lo que se observa es la conformación de un campo conflictivo por la construcción de una nueva identidad. Nuestra perspectiva sostiene que la fortaleza de este movimiento social radica en la constitución de una identidad compartida que, a su vez, deriva de manera importante de una visión colectiva sobre el significado del territorio.

\section{¿Modelo de desarrollo en crisis?}

El modelo de desarrollo es una particular combinación de factores políticos, económicos, sociales y culturales. Más específicamente, de tres dimensiones (Arocena, 1995):

- Paradigma tecnológico o modelo de industrialización: se refiere a los principios que guían las formas que adoptan la organización y división del trabajo.

- Régimen de acumulación: describe las compatibilidades e interrelaciones entre las condiciones de producción y las de consumo y distribución del producto social. El régimen de acumulación se puede considerar como el resultado macroeconómico del funcionamiento del modo de regulación correspondiente a determinado paradigma tecnológico.

- Modo de regulación político y social que debe asegurar la adecuación de los comportamientos dentro del régimen de acumulación, y que se lleva a cabo tanto a través de restricciones institucionales como de patrones culturales y éticos.

En cada una de estas configuraciones, es posible reconocer un conjunto de sujetos a cargo de la conducción política del modelo.

En los últimos dos tercios del siglo XX, en Chile, se desplegaron dos modelos de desarrollo: uno que denominaremos estadocéntrico y otro de carácter neoliberal. Cada uno de ellos posee características específicas. Por un lado, el modelo desarrollista desplegado en América Latina se caracterizó por ser fuertemente paternalista, con un Estado intervencionista, por poseer un modelo económico proteccionista y a su vez extractivista, centrado en la industrialización por sustitución de importaciones. Por otro lado, un modelo neoliberal primario exportador, reforzador de la privatización de las empresas como de los servicios sociales, con un Estado encargado de garantizar el crecimiento económico como puerta de entrada a la propiedad privada, capitalización, 
consumo, etc., y una política de apertura al comercio exterior, a partir de la ampliación en los márgenes de ganancia de la empresa (Ffrench-Davis, 2005; Oszlak, 1997; Garretón, 2000; Muñoz, 1992; BM, 1997; Tomassini, 1995).

Sin embargo, a pesar de las disparidades, ambos modelos de desarrollo comparten tres elementos: 1) sistema de toma de decisión centralizado, ${ }^{1}$ 2) modelo económico extractivista y 3) ausencia de preocupación por el medio ambiente. A partir de cada eje, podemos observar el surgimiento del nuevo modelo que se está constituyendo en Aysén.

Ahora bien, el punto de encuentro entre los dos modelos de desarrollo en Chile son la explicación del por qué los temas de descentralización y valoración por la iniciativa local han cobrado relevancia en el último tiempo. Esto, siguiendo a Arocena (1995), se debe a que la noción misma de desarrollo, y de las diversas perspectivas predominantes, han sido puestas en tela de juicio a partir de la crisis de los modelos de desarrollo.

Con el término de la Segunda Guerra Mundial, surgieron las primeras nociones de desarrollo dirigidas, principalmente, hacia los países del tercer mundo. El desarrollo, por tanto, no debía ser producto de una construcción colectiva. Por el contrario, provendría de una evolución por la cual aquellos países dependientes de los centros industriales lograrían ser una sociedad industrializada. Asimismo, desde el estructuralismo, se sostenía que los países tercermundistas debían superar el problema centro-periferia, para lo cual era urgente realizar cambios estructurales que implicaran la modificación de la propiedad, estructura productiva y distribución territorial.

Las primeras nociones de desarrollo dejaron al descubierto sus falencias, específicamente con la crisis del Estado desarrollista. Según el diagnóstico de los estructuralistas, la crisis se debió a diversos motivos: por una parte, a la fuerte dependencia que tenían los países periféricos hacia los centros, el obstáculo de la gran hacienda y el consiguiente deterioro de la vida del campesinado, acostumbrado y dedicado a economías de subsistencia. También a la falta de acceso a la propiedad por parte del campesinado, que generaba pobreza y carencia de oportunidades - especialmente en los países andinos y centroamericanos- y a su vez el bajo poder adquisitivo de la población latinoamericana que impedía la absorción industrial, al no existir quién consumiera los productos que eventualmente se podrían manufacturar.

A partir de la crisis del modelo desarrollista -que coincidió con la crisis de la democracia en América Latina- se comenzó a desplegar un segundo modelo de desarrollo de corte neoliberal. En el caso chileno, el nuevo modelo fue llevado a cabo por el régimen autoritario a partir de 1973 y se caracterizó por abandonar la idea de Estado intervencionista, para pasar a uno de carácter desregulado y liberalizador, débil respecto

\footnotetext{
${ }^{1}$ Bucciarelli (2009) resalta el carácter dependiente del poder central que tuvo la Patagonia argentina, así como ocurrió con el caso chileno, que consideró la meseta patagónica solo como un espacio para la conquista, por lo cual las divisiones administrativas fueron privadas de autonomía.
} 
de su espacio de acción, con una economía primaria exportadora y de libre mercado, donde la privatización fue uno de sus principales motores.

Sin embargo, este modelo entró en cuestionamiento con la ola democratizadora de América Latina e iba a dejar en evidencia sus falencias: las grandes desigualdades en cuanto a obtención de recursos, una idea de modernización vacía donde la tecnología avanzaba a ritmo apresurado mientras el bosquejo social reflejaba disparidades, el alto centralismo y el descuido por los niveles locales, incluso considerando los incipientes proyectos descentralizadores que tuvo el régimen autoritario.

Los cuestionamientos al modelo neoliberal abrieron, paulatinamente, un espacio para repensar los modos de desarrollo. El concepto de desarrollo alternativo tomó fuerza a fines del siglo XX, insertando la idea de desarrollo local como parte de las nuevas propuestas que contemplaron dos problemas no trabajados por los modelos de desarrollo desplegados en Chile: el centralismo y el descuido por el medio ambiente. ${ }^{2}$

Pese a los cuestionamientos que experimenta el modelo neoliberal, este sigue predominando como modelo de desarrollo en Chile, y aunque ya no es autoritario en lo político, continúa siendo extractivista en lo económico y descuidado en lo que respecta a la dimensión medioambiental. Contra ese esquema de acumulación por desposesión (Harvey, 2004) es que protestan las sociedades territoriales. ${ }^{3}$

$\mathrm{Al}$ perpetuar el extractivismo sin establecer normas que fomenten el cuidado por el medio ambiente, y al ser Chile un Estado centralista - propio de ambos modelos de desarrollo- de carácter unitario, con un presidencialismo hipercentralizado y un sistema de partidos nacionalizado, lo que se comienza a gestar es un malestar social materializado en diferentes movimientos sociales: ambientalistas, territoriales, estudiantiles, etc. Dicho malestar es el que está a la base del movimiento en Aysén, impulsado por responder y conformarse como un modelo de desarrollo diferenciado, el cual busca reivindicar el desarrollo local con una idea de identidad particular basada en la apropiación de significado de su propio territorio.

\section{Desarrollo local e identidad}

Los cuestionamientos al modelo de desarrollo han dado lugar a nuevas perspectivas que, más allá de las diversas nomenclaturas (endógeno, sustentable, etc.), pone de relieve la importancia de la dimensión local. No solo por la relevancia de la "pequeña escala",

\footnotetext{
2 Según Núñez, Aliste y Bello (2014), una vez finalizada la dictadura en Chile, surgen los discursos desarrollistas incitados desde la valoración del turismo, la protección de la naturaleza, que otorgan relevancia al sector y dan paso a un discurso ambientalista y sustentable.

${ }^{3}$ Harvey reinterpreta el tradicional concepto de acumulación originaria de Marx, para sostener que la violencia, la depredación y el engaño son prácticas permanentes del proceso de acumulación capitalista que no se circunscriben a una etapa fundacional.
} 
sino, y fundamentalmente, por los procesos de constitución de nuevos sujetos sociales que se despliegan en el territorio.

Siguiendo a Larraín (2005), la identidad es un proceso de construcción y reconstrucción de una narrativa de sí mismo en un proceso social mediado por símbolos:

\begin{abstract}
Un significado más adecuado de identidad deja de lado la mismidad individual y se refiere a una cualidad o conjuntos de cualidades con las que una persona o grupo de personas se ven íntimamente conectadas. En ese sentido la identidad tiene que ver con la manera en que individuos y grupos se definen a sí mismos al querer relacionarse -identificarse - con ciertas características. (p. 23)
\end{abstract}

Por otro lado, Arocena (1995) plantea la importancia de la identidad en los procesos de desarrollo local como un elemento clave en el alcance de su éxito, argumentando que la construcción de la identidad es producto de un proceso de acumulación cultural que implica continuidades y cambios a lo largo de la historia.

La identidad local se define en función de la pertenencia al territorio, como espacios donde los grupos humanos habitan y desarrollan sus ritos, valores, creencias y costumbres en una línea de tiempo dinámica que implica ausencias y permanencias. El territorio cumple el papel de palanca de desarrollo que demanda la movilización de los actores humanos, recursos propios con una proyección hacia el futuro.

Para este mismo autor, la construcción de identidades colectivas demanda individuos, grupos e instituciones como actores sociales que, a través de la acción territorial en el campo de lo político, económico, social y cultural, impulsen el desarrollo local. La presencia de actores-agentes de desarrollo local será fundamental en el éxito de los procesos de desarrollo en la medida en que cuide el medio natural, produzca iniciativas de interés local y potencien las estrategias de producción.

En su relación con el territorio, los grupos se apropian de él y generan, no solo su utilización para la satisfacción de sus necesidades materiales, sino significados que aportan a la constitución de su identidad:

Se entiende por territorio el espacio apropiado por un grupo social para asegurar su reproducción y la satisfacción de sus necesidades vitales, que pueden ser materiales o simbólicas [...]; el proceso de apropiación sería entonces consubstancial al territorio. Este proceso marcado por conflictos, permite explicar de qué manera el territorio es producido, regulado y protegido en interés de grupos de poder. (Giménez, 2005, p. 9)

La apropiación del territorio puede ser utilitaria y funcional (mercancía generadora de renta, fuente de recursos, de control militar e importancia geopolítica o como 
jurisdicción político-institucional), o simbólico-cultural cuando el énfasis está puesto en su consideración como lugar de historia, tradición, reserva ecológica, o ambiental referente de identidad del grupo, o símbolo-metonímico. La región

puede ser apropiada subjetivamente como objeto de representación y de apego efectivo y, sobre todo, como símbolo de identidad socioterritorial. En este caso, los sujetos (individuales y colectivos) interiorizan el espacio regional integrándolo a su propio sistema cultural. Con esto hemos pasado de una realidad territorial "externa", culturalmente marcada, a una realidad territorial "interna" e invisible, resultante de la filtración de la primera, con la cual coexiste. (p. 17)

En este contexto, la identidad puede ser concebida como el recurso, la potencialidad y la dinámica mediante los cuales los colectivos humanos se transforman en sujetos de acción en contextos específicos:

La identidad es, más bien, el proceso mediante el cual los colectivos humanos reconstruyen, permanentemente, la coherencia y consistencia de sus orientaciones mutuas en el espacio en tensión definido por lo que han sido y por lo que quieren o necesitan ser en los nuevos contextos. (Güell, 1996, p. 61)

De acuerdo con Larraín, la identidad "es un proceso de construcción en la que individuos y grupos van constituyendo un discurso sobre sí mismos en estrecha relación con otras personas y grupos. La construcción de la identidad es así un proceso social en un doble sentido" (2005, p. 115), tanto porque su identidad alude a categorías sociales y culturalmente compartidas (religión, género, clase, nacionalidad, territorio, etc.) como porque esta se refiere a "otros" cuyas expectativas internalizamos y de los cuales deseamos diferenciarnos.

Cualquiera que sea el modelo de desarrollo, las posibilidades de su despliegue y consolidación dependen de manera significativa de la identidad que logren constituir los sujetos. Esa identidad establece un recurso fundamental para el impulso de un modelo de desarrollo, más aún cuando se propone diferenciarse del esquema predominante.

\section{Desarrollo, territorio e identidad en Aysén}

Miryam Chible resalta una identidad regional fuerte:

Somos gente de los espacios libres, sin las rejas del Valle Central, con praderas, hielos y bosques, donde se baila chamamé, se juega al truco y se capea el viento con un asado de cordero al palo y chimichurri. Somos las regiones que estamos prohibiendo el plástico y 
amamos nuestra tierra virgen, uno de los cinco ecosistemas casi intocados de la tierra. ${ }^{4}$

(entrevista con Miryam Chible, 6 de septiembre de 2014)

El metarrelato de la líder patagónica aysenina se hace parte de los regionalismos y protonacionalismos que en la diferenciación del otro (en este caso, los chilenos del valle central) busca los elementos para la construcción de nueva identidad.

La renovada identidad patagónica de una región aislada y despoblada como Aysén puede devenir tanto una macrorregión sustentada en un movimiento protonacionalista patagónico o, al menos, una creciente demanda de mayor autonomía. Como bien explica Núñez Seixa (1999), en el caso español, la demanda de poder territorial con soberanía estuvo polarizado entre los "primordialistas" del jus sanguinis y los "constructivistas" de la Revolución francesa (que construyen una comunidad política elegida por la vía del fortalecimiento de su propia estatalidad).

La demanda de autonomía y devolución de recursos de los tres movimientos regionalistas fuertes chilenos (el nortino por renta minera, los mapuches y el patagónico aysenino) apuntan en esta segunda dirección (Marimán, 2012; Penaglia y Valenzuela, 2014). Puede no llegar a constituirse en nación, pero son muestras de lo que Saz Campos y Romeo Mateo (2004) llaman un "regionalismo vital”.

Los estudios transfronterizos en América Latina (Tapia y González, 2014) dan cuenta de esta posibilidad de construir, conjuntamente, la identidad étnica, el regionalismo fuerte y lo nacional, en aquello que Beck y Grande (2006) llaman un nacionalismo cosmopolita y que permite que, por ejemplo, una misma persona pueda ser catalán/ español/europeo o, en nuestro caso, aysenino/chileno/patagónico. ${ }^{5}$

La revisión del conflicto en Aysén y su itinerario nos aporta evidencia para una mejor comprensión del proceso. Aysén comenzó a inicios de 2000 a movilizarse contra proyectos de instalar plantas de explotación de las minas de zinc. Luego, emprendió

\footnotetext{
${ }^{4}$ Miryam Chible, hija de colonos sirios que llegaron a Aysén a inicios del siglo XX, es líder del movimiento social Patagonia sin Represas que se opone a la instalación de grandes hidroeléctricas en el río Baker (entrevista, 6 de septiembre de 2014).

${ }^{5}$ Tal como lo analiza Rojas (2013), la mirada de Aysén es distinta de la de Magallanes o Neuquén, con poder y tradición en ambos países, que incluye representación política propia. En Neuquén, el movimiento Popular Neuquino gobierna en todos los periodos democráticos desde 1958, y en Magallanes tuvieron al Partido Regionalista como fuerza principal en las décadas de 1930 y 1940. La región de Magallanes posee una fuerte memoria histórica de lucha social marcada por hitos, como el movimiento obrero que terminó con la Matanza de la Federación Obrera en 1920 como un hito histórico que caracterizó la época de la Patagonia rebelde (Bayer, 2009), la creación del primer partido regionalista magallánico en 1931 y una de las primeras protestas contra la dictadura de Pinochet (el puntarenazo) en febrero de 1984. Junto con ello, la identidad magallánica -manifestada a través de distintos diacríticos sociales, como la bandera y el himno- se gesta desde un clima adverso (en palabras de magallánicos: "la lucha contra la naturaleza, el viento y frío") y de la interacción de migrantes chilotes, croatas, italianos, ingleses y argentinos. A su vez, el aislamiento -inexistencia de conectividad terrestre con Chile- les ha llevado a generar un mayor vínculo con Argentina (entre los municipios de Aysén y Chubut), compartiendo una identidad común patagónica con fuerte interdependencia económica y social.
} 
una larga resistencia contra un megaproyecto de represas en el río Baker impulsado por el Grupo Matte. El proyecto fue finalmente desechado en 2014 por el Gobierno de Michelle Bachelet tras un conjunto de movilizaciones que incluyó, entre otras acciones, la toma de dos de las principales ciudades de la región (Coihaique y Aysén) en 2011.

El movimiento social demandaba atención del Estado central y rechazo a las hidroeléctricas. Con una imagen de su territorio contaminado por tendidos eléctricos para alimentar Santiago y la minería del norte, los ayseninos apoyaron masivamente el movimiento Patagonia sin Represas. En la lucha, se unieron las comunidades locales, empresarios turísticos, el senador de centro-derecha ecologista Horvarth, el obispo Infanti y el empresario norteamericano Douglas Tompkins, partidario de la ecología profunda y filántropo ambiental que donó cientos de miles de hectáreas para hacer megaparques nacionales en el norte y sur de la región de Aysén.

El conflicto en Aysén se desata en un contexto de baja en la industria del salmón y de la pesca. Esto provocó el malestar de los pescadores que, junto con dirigentes de la zona, el 7 de febrero de 2012 se tomaron el puente Presidente Ibáñez, principal acceso a puerto Aysén por vía terrestre. Seis días más tarde, alrededor de sesenta pescadores del archipiélago de las Guaitecas se tomaron el aeródromo de Melinka, con lo cual mostraron su profundo rechazo a la ley de pesca y exigieron el alza de las cuotas de pesca y la presencia de la intendenta de Aysén, Pilar Cuevas, y del subsecretario de Pesca, Pablo Galilea.

Luego de este suceso, distintas localidades de la región fueron simpatizando con el movimiento y sumándose a la lucha: en Caleta Andrade, los habitantes se tomaron el aeródromo, en Coyhaique hubo manifestaciones durante la noche (un grupo aproximado de doscientas personas salieron a las calles levantando barricadas en distintos sectores de la ciudad tras lo cual se produjeron apagones). Para el 17 de febrero casi la totalidad de la región estaba movilizada. La ruta que une Villa Mañihuales con Puerto Aysén estaba bloqueada; el cruce del camino de Coyhaique-Chile Chico y Cochrane fue bloqueado por los habitantes de Puerto Guadal; y la carretera hacia Puerto Chacabuco seguía con barricadas.

En distintos sectores de la región, como en Chile Chico, se realizaron marchas con las cuales manifestaron su solidaridad con la movilización de Puerto Aysén, que se extendieron también a Punta Arenas y Santiago.

Entre las demandas más importantes que desarrolló la asamblea ciudadana y el movimiento social por Aysén, que agrupa a diversas organizaciones sociales, podemos destacar las siguientes: rebaja sustancial del precio de los combustibles (leña, gas, petróleo, parafina, bencina), salud de calidad, equidad laboral, participación ciudadana vinculante, empoderamiento de la pesca artesanal regional, subsidio al transporte e integración física, regionalización de recursos naturales y universidad estatal regional.

El conflicto de Aysén evidencia un proceso de construcción de un sujeto plural y transversal. En este contexto, una ciudadanía postergada en cuanto a prestaciones de 
salud, educación y costo de la vida convergió con organizaciones sindicales movilizadas debido a la coyuntura particular de la disminución en las cuotas de pesca artesanal; con el trabajo de redes trasnacionales de defensa ambientales como Patagonia sin Represas (que durante 2011 había generado movilización en distintas partes de Chile contra el proyecto Hidroaysén que reactivó el tejido social), sumado a la presencia de actores tradicionales como la Central Unitaria de Trabajadores y la Asociación Nacional de Empleados Fiscales.

A lo largo del desarrollo del conflicto, los diversos actores fueron construyendo un metarrelato que tiene como eje estructurante la defensa de "un paraíso intocable”. Se trata de un conflicto que va más allá de los NIMBY (not in my backyard), más espontáneos y de corto plazo, para situarse en el ámbito de los NIABY (not un anybody's back yard), de politización ascendente que les llevó a organizarse y lograr elegir uno de los dos diputados (Iván Fuentes, líder de los pescadores) y que el Gobierno rechazara el megaproyecto de la represa.

Siguiendo a Smith (1991), crearon un etnosimbolismo. Un híbrido entre el primordialismo (Aysén tierra pura de los patagones/tehuelches) y el modernismo (la ecología y el ecoturismo como destino común) recurriendo al uso de los medios modernos y al marketing para construir su macroterritorio (Gellner, 1988). Nace, entonces, una épica (contra Santiago y el extractivismo) y una lírica (el paraíso patagónico ecologista) que recrea una macrorregión o protonación de comunidades construidas e imaginadas en la clásica definición de Anderson (1993): la recreación de "comunidades imaginadas" por grupos de interés que se convierten en agentes culturales, posibilitada por los nuevos medios tecnológicos.

Lo notable del caso aysenino es la alianza interclases sociales que suponen todos los casos de regionalismos fuertes o "nacionalismos pequeños", como señala Hroch (2001). Siempre el sentimiento de identidad se activa en la conciencia de ser distintos del otro, producto de los viajes, el comercio o la amenaza. El dirigente nacional de los periodistas chilenos y dirigente del movimiento social de Aysén, Patricio Segura, lo manifiesta así: "La invasión de represas y sus tendidos eléctricos era impensable para los antiguos colonos y para los nuevos colonos como en mi caso, que opté por la Patagonia desde la militancia ecológica. No nos podían cambiar nuestro propio país y territorio" (entrevista con Patricio Segura, 20 de abril de 2015). De esta manera, el regionalista transpatagónico de Aysén se resiste al nacionalismo banal (Billig, 2006) chileno que liga la idea del desarrollo a la industria, la minería, la explotación del gas y del petróleo y las represas. ${ }^{6}$

\footnotetext{
${ }^{6}$ La identidad que se activa producto de una amenaza concierne bastante con el cuidado por el medio ambiente y la defensa del territorio en contra de la explotación de los recursos naturales por parte de las empresas. Núñez, Matossian y Vejsbjerg (2012) recalcan la importancia de los parques nacionales para la Patagonia argentina, poniendo de relieve el fomento que tuvo para la producción económica en la región la producción ganadera y la explotación de madera.
} 


\section{El sentido macrorregional patagónico en Aysén}

La Patagonia como territorio, identidad e imaginario se expande en un proceso en el que el poder de las capitales nacionales disminuye y crecen las regiones (Naím, 2013). Su protagonismo crece a la par de la identidad del Caribe y el "mundo maya”, junto a la Amazonia, la Orinoquia, lo "andino inca”, para concluir en la impoluta y turística Patagonia, como la otra gran macrorregión marcada de relaciones transfronterizas que fluyen en pugnas en América Latina más allá de la estatalidad nacional (Tapia y González, 2014).

La Patagonia fue un territorio en disputa entre Argentina y Chile (Navarro, 2005). Esta disputa solo se resuelve con acuerdos a fines del siglo XIX en que Chile se concentra en el norte y acepta que la zona de pedregales y tundras - sin gas ni petróleo descubierto y valorado - sea parte de Argentina que había iniciado pioneramente su colonización con comunidades de Gales que introducen las ovejas y la industria lanar, junto con la producción frutícola en el alto valle del río Negro, para luego ser el foco de los Gobiernos desarrollistas por su ingente riqueza en hidrocarburos y la generación eléctrica argentina por la obra de El Chocón como una de las más importantes fuentes hidroeléctricas del último tercio del siglo XX (Bridges, 1952; De Agostini, 1956).

Desde entonces, Chile se concentró en su región de Magallanes como polo de desarrollo en el estrecho de Magallanes, con una nutrida colonia inglesa y croata, además de chilenos de origen de Chiloé (chilotes), donde se combinó la zona de servicios del puerto transoceánico antes de la apertura del canal de Panamá, la dinámica industria ganadera, petróleo y turismo con proyección antártica (Lamig, 1957; Martinic, 1977; Emperaire, 2002).

Hoy, las demarcaciones territoriales sobre la Patagonia son difusas. Por el auge turístico de la marca Patagonia, las zonas incluso de la parte sur de la provincia de Buenos Aires y de Mendoza se consideran el inicio de la Patagonia. En el caso chileno, en Puerto Montt, se creó en 2000 entre intelectuales de izquierda y derecha el llamado Club de la Patagonia, que a juicio de Patricio Cantos "encierra nuestra pertenencia al mundo austral que viene después del territorio mapuche. Acá en Puerto Montt y el archipiélago de Chiloé se inicia el mundo de la Patagonia” (entrevista con Patricio Cantos, 2 de diciembre de 2014).

En el estudio de Gonzalo Soto (2015),7 quien entrevistó a un centenar de líderes sociales, funcionarios y políticos sobre el regionalismo aysenino en comparación con sus regiones aledañas (Los Lagos [Puerto Montt] y Magallanes [Punta Arenas], se comprueba que Aysén es la región con mayor disposición a fusionarse en una megarregión patagónica. Si bien existe diversidad en la propia demarcación de la Patagonia, queda claro el interés de los aysesinos por una delimitación macrorregional o protonacional (tabla 1).

\footnotetext{
${ }^{7}$ Este apartado recoge el análisis realizado por Soto (2014) dentro de su tesis de grado.
} 
Tabla 1. ¿Cuál cree que es la delimitación de la Patagonia chilena?

\begin{tabular}{|c|c|c|c|c|c|}
\hline & & 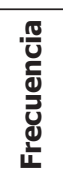 & 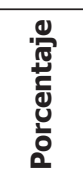 & 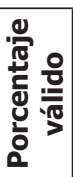 & 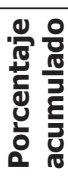 \\
\hline & Región de Araucanía a región de Magallanes & 2 & 1.9 & 1.9 & 1.9 \\
\hline & Región de Los Ríos a región de Magallanes & 2 & 1.9 & 1.9 & 3.8 \\
\hline & Región de Los Lagos a región de Magallanes & 5 & 4.6 & 4.8 & 8.7 \\
\hline & Provincia de Llanquihue a región de Magallanes & 4 & 3.7 & 3.8 & 12.5 \\
\hline & Desde Cochamó, provincia de Palena a región de Magallanes & 4 & 3.7 & 3.8 & 16.3 \\
\hline Vólidor & Chiloé, región de Aysén y región de Magallanes & 4 & 3.7 & 3.8 & 20.2 \\
\hline Vallados & Desde Puerto Montt a región de Magallanes & 16 & 14.8 & 15.4 & 35.6 \\
\hline & Provincia de Palena y región de Aysén & 1 & 0.9 & 1.0 & 36.5 \\
\hline & Provincia de Palena a región de Magallanes & 49 & 45.4 & 47.1 & 83.7 \\
\hline & Región de Aysén y región de Magallanes & 15 & 130.9 & 14.4 & 98.1 \\
\hline & Solo región de Magallanes & 2 & 1.9 & 1.9 & 100 \\
\hline & Total & 104 & 96.3 & 100 & \\
\hline Perdidos & NS/NR & 4 & 3.7 & & \\
\hline Total & & 108 & 100 & & \\
\hline
\end{tabular}

Fuente: Elaboración según Soto (2014).

Nota: Los grupos mostrados en la tabla se establecieron de acuerdo con las tendencias establecidas en el desarrollo del instrumento. Se agruparon para facilitar el análisis. Es importante clarificar que, en el caso de la provincia de Palena, se dieron ciertas discrepancias principalmente marcando como inicio la comuna de Chaitén, Hualaihué y, en menor medida, Futaleufú.

Así es como la opción que recoge la mayoría (47.1 \%) de las preferencias identifica la Patagonia con el territorio de las regiones de Magallanes y Aysén más la provincia de Palena. El segundo grupo piensa que la Patagonia se extiende desde Puerto Montt a Magallanes (15.4\%), mientras que otro 14.4 \% la circunscribe solo a Aysén y Magallanes.

Los que sostienen que la Patagonia comienza más al norte exponen sus argumentos. Diego Benavente manifiesta que "la Patagonia o Puel Mapu para el mundo Mapuche comienza desde las alturas de la Araucanía (Temuco) y tiene una relación muy estrecha con el Lafken Mapu" (entrevista con Diego Benavente, 6 de septiembre de 2014). Por su parte, Leopoldo Pineda considera que la Patagonia chilena se extiende desde el Lago Villarrica hasta el fin del continente (entrevista con Leopoldo Pineda, 6 de septiembre de 2014).

Carlos Castaing propone una interpretación sobre el porqué de esta ampliación:

A efectos de la promoción turística del sur de Chile, la tendencia es situar la frontera patagónica lo más al norte posible. Algunos hablan del río Biobío, la misma altura del río Negro que delimita la frontera "turística” de la Patagonia argentina. En la Araucanía, Pucón y Villarrica se promocionan como Patagonia. En mi opinión, la Patagonia como entidad cultural comienza en Palena. (entrevista con Carlos Castaing, 6 de septiembre de 2014). 
En esta misma línea, el diputado Morano es enfático en su opinión al sostener que la delimitación de la Patagonia está definida legalmente y coincide con las regiones de Aysén y Magallanes (tal vez Palena):

Puerto Montt al norte, no puede pretender ser considerada parte de ella solo por el objetivo propagandístico... porque ya hay una marca conocida mundialmente Patagonia y, por tanto, ahí se trabaja esa marca, no el espíritu de la Patagonia, no la vivencia de la Patagonia, no al sufrir de la Patagonia. Hay una Patagonia que se llamó Patagonia rebelde, que se llena de los movimientos sociales en Argentina en los años veinte. Puerto Montt no es parte de eso, Valdivia no es parte de eso... Podrán estar muy orgullosos de lo que hicieron los pueblos originarios, los mapuches, pero no de los patagones que no vivieron ahí. (entrevista con Juan E. Morano, 6 de septiembre de 2014).

Similar es la percepción de David Sandoval, diputado por la región de Aysén, quien resalta: "La gente de la Décima Región fue muy mal alumna de geografía. Lo que ellos buscan con el tema de asociarse a la Patagonia es solamente un fin comercial. Puerto Montt no tiene nada que ver con la Patagonia" (entrevista con David Sandoval, 6 de septiembre de 2014).

Pese a ello, las autoridades locales de Puerto Montt defienden la ciudad como parte de la Patagonia. El alcalde de la ciudad afirma:

\footnotetext{
Hay una discusión muy extensa respecto de la Patagonia, dónde comienza y cuáles son sus límites. Me atrevería a precisar que comienza en la zona de la provincia de Llanquihue por el norte hasta el Cabo de Hornos por el sur. Para nosotros, Puerto Montt es la "puerta de entrada a la Patagonia” austral. De hecho, en la nueva imagen corporativa de la ciudad dice: "Puerto Montt... Patagonia Chile". Además, hemos hecho un convenio con los municipios de Coyhaique y Punta Arenas, precisamente para potenciar esa marca. (entrevista con Gervoy Paredes, 6 de septiembre de 2014)
}

Esta información sería complementada por la que entrega el concejal de la comuna de Puerto Montt, Fernando España: "Hoy se trabaja para que la Patagonia comience en la región de Los Lagos, específicamente en Puerto Montt” (entrevista con Fernando España, 6 de septiembre de 2014).

En opinión de la diputada Marisol Turres, la Patagonia "comienza en el kilómetro 1 de la carretera Austral, es decir, en Puerto Montt y continúa hasta Magallanes. Incluye a mi juicio la comuna de Cochamó" (entrevista con Marisol Turres, 6 de septiembre de 2014).

Una iniciativa importante en el ámbito municipal es la impulsada por un grupo de autoridades locales que, liderada por la comuna de Tortel, se encuentra trabajando en 
la creación de la Asociación de Municipios de la Patagonia, que tendría el objetivo de elaborar una agenda para el desarrollo desde los mismos territorios. A efectos de la asociación, estarían contemplados los municipios de la provincia de Palena y los de las regiones de Aysén y Magallanes. Al ser un tema de asociación de municipios, se convierte en un asunto político, lo cual muchas veces, como en la mayoría de las convergencias municipales, se transforma en una traba para su desarrollo.

Los consejeros regionales también tienen dos propuestas. Por un lado, la creación de una asociación de consejeros de la Patagonia: "Como consejero regional, estamos en un trabajo... en una asociación de consejeros de la Patagonia considerando Los Ríos, Los Lagos, Aysén y Magallanes y también en convenios con la Patagonia argentina para lograr el mayor desarrollo de nuestros pueblos" (entrevista con Julio Uribe, 6 de septiembre de 2014). Por otro lado, en el Congreso, se creó la bancada de la Patagonia, compuesta por la senadora Carolina Goic, el senador Patricio Walker y los diputados Juan Morano e Iván Fuentes, quienes tendrían una delimitación de la Patagonia como bancada correspondiente a las regiones de Aysén y Magallanes.

Como se puede apreciar en la tabla 1, la mayoría de las respuestas se consideran dentro de la provincia de Palena, y las regiones de Aysén y Magallanes. Ciertas opiniones, principalmente de dirigentes de la región de Magallanes, expresan un fuerte sentido de pertenencia a la Patagonia chilena, al expresar, "para mí comienza al revés de muchos, en Cabo de Hornos y debiese de terminar en Palena".

Si bien este tipo de repuesta no fue mayoritaria dentro de los dirigentes políticos, sociales y académicos de la región de Magallanes, sí se presentaron en más de una ocasión, y dejaron entrever el fuerte arraigo a la Patagonia (aunque en casos especiales, siempre se expresó el concepto de magallánico), precisamente, por ser la zona donde se habrían dado los primeros reconocimientos y por la importancia que tuvo Punta Arenas en su condición de puerto libre - quitado en 1912- y su importancia geopolítica y estratégica que favoreció el comercio en la zona e incluso generó vanguardia dentro de la misma Patagonia argentina.

Por tanto, según lo reflejado tanto en la tabla 1 y en relación con el marco teórico utilizado en la investigación, habría una tendencia mayoritaria por lo expresado en los trabajos de Martinic (1977) a ubicar el comienzo de la Patagonia chilena en el seno de Reloncaví. No obstante, se presentaron diferencias entre los consultados sobre este punto, ya que algunos lo consideran desde Puerto Montt, la bahía de Puerto Montt o la comuna de Cochamó.

Cabe destacar que muy pocos de los consultados tenían conocimiento previo del estudio realizado por la Universidad de Chile por encargo del Ministerio de Bienes Nacionales. Sin embargo, hubo aceptación - en menor medida que en los casos con porcentajes más elevados- de dicho estudio. Finalmente, frente a lo que concierne del tratado limítrofe de 1881, solo tres consultados lo utilizaron para justificar sus respuestas. 
Los datos recogidos en la encuesta ante la pregunta “¿Cree que exista una identidad patagona?" evidencian una clara respuesta positiva: $89.7 \%$ de los consultados considera que sí existe una identidad patagónica (tabla 2). Dicha identidad patagona se expresa en las costumbres del baqueano, el gaucho, el folclore, la vestimenta, los modismos propios de la zona y la fuerte relación transversal este-oeste entre los habitantes de Chile y Argentina. Por su parte, las características geográficas y las inclemencias del tiempo marcan un estilo de vida propio, vivir durante un solo día "las cuatro estaciones". En cuanto a los procesos de colonización que se vivieron dentro de los territorios que comprenden la Patagonia, se componen de características muy similares que marcan su gran heterogeneidad de culturas que los poblaron, reconociendo gran importancia de la cultura chilota. Otra característica muy presente son las condiciones de aislamiento y abandono desde el Gobierno central.

Algunas opiniones que recogen estas impresiones sobre la existencia de una identidad patagónica son las expresadas por el senador Horvath:

Sí, hay una correspondencia cultural con un territorio único, que es más conocido a nivel internacional que Chile. Su cultura es una mezcla entre colonos que vienen de Chiloé y distintas áreas y grupos de extranjeros, lo cual genera una identidad única. Es el gaucho chilote y algo más, que sin duda genera algo propio. (entrevista con Antonio Horvath, 6 de septiembre de 2014)

A la vez, podemos apreciar características distintas de las de la zona centro-sur del país, expresadas claramente por Elías Muñoz, exjefe de Prensa de Radio Santa María:

[El patagón] gusta del mate, de la carne y poco de las verduras y frutas. Está mirando al resto del país (desde Puerto Montt hacia el norte) como otra nación, se siente poco integrado a Chile, ello explica el nacimiento de banderas propias en Aysén y Magallanes... es desconfiado del nortino, que para el patagón es quien viene desde Puerto Montt hacia el norte. (entrevista con Elías Muñoz, 6 de septiembre de 2014).

Siguiendo esta línea, pero en un registro más político, Juan Arcos, concejal de la comuna de Punta Arenas, plantea:

Para mí la Patagonia per se es una nación donde quienes habitamos tenemos una identidad cultural. Además de ser una colonia susceptible al tenor de las resoluciones 1514 y 1541 de las Naciones Unidas sobre las colonias, de votar ya sea su autonomía como estado asociado a Chile, a otra potencia o como un país independiente. (entrevista con Juan Arcos, 6 de septiembre de 2014) 
Sin embargo, es muy importante recalcar que dentro del territorio de la Patagonia chilena no existe solo una identidad. Esta percepción queda bastante clara en el análisis de Ximena Urbina, profesora del Instituto de Historia de la Universidad Católica de Valparaíso y vinculada a la Patagonia a través de dos proyectos del Fondo Nacional de Desarrollo Científico y Tecnológico (números 3090054 y 1120704), quien expresa en cuanto a la identidad patagona:

\begin{abstract}
Creo que en los últimos años se ha construido una identidad patagónica. Esta identidad se ha construido asociada a la historia, específicamente a la colonización de las pampas, la figura del gaucho, el mate y el truco. Esta identidad construida ha gustado en la Patagonia, porque identifica con lo argentino, lo foráneo y lo no indio. Sin embargo, la realidad histórica costera o de los canales es más antigua (desde los canoeros australes y la proyección desde Chiloé en el periodo colonial), pero no ha sido valorada por remitir a lo chilote y lo indígena. Existe una identidad patagónica bordemarina, pero es considerada como de menor categoría frente a la pampeano-colona. (entrevista con Ximena Urbina, 6 de septiembre de 2014)
\end{abstract}

Por otra parte, entre quienes consideran que no existe una identidad patagónica, la mayoría se concentra en el grupo "municipios de Aysén”. En sus respuestas, se rechaza la idea de una identidad propia, porque, a su juicio, la identidad requiere la creación de nuevos elementos y no solo de apropiarse de elementos de culturas distintas.

El concejal de la comuna de Cochrane, Rodrigo Rivera, afirma:

No creo que tengamos una identidad propia patagónica, creo que ha sido una mezcla de las culturas y la influencia del pueblo argentino lo que nos ha marcado más con nuestras costumbres y tradiciones, por tener más facilidad de acceso a Argentina que a nuestro mismo país. Me gustaría que la región de Aysén pueda tener una identidad propia como la tienen la región de Magallanes donde sí son una república independiente, creo que en ese sentido los ayseninos somos muy malos para organizarnos tratando cada uno poder llevar a cabo sus propios ideales y no viendo el bien colectivo. (entrevista con Rodrigo Rivera, 6 de septiembre de 2014)

La segunda opinión en concordancia con lo mencionado es de Sandra Planzer, dirigente social de la región de Aysén, quien comenta que "una identidad propia no existe. A través de la historia nos hemos estado empapando de las costumbres patagónicas argentinas. Es lamentable decirlo, pero así es. La mayoría de los que vivimos aquí sabemos bailar chamamé pero no cueca, el tema de las domaduras, el hecho de andar vestidos de gaucho, etc. (entrevista con Sandra Planzer, 6 de septiembre de 2014)

Esta opinión se complementa con la de Marisol Turres, diputada de la región de Los Lagos: "Creo que a diferencia de la Patagonia argentina, no hemos definido 
símbolos o características de la Patagonia chilena” (entrevista con Marisol Turres, 6 de septiembre de 2014).

Algunos, si bien reconocen la existencia de una identidad patagónica, ella no coincide con el territorio que se identifica como Patagonia. Esto lo apreciamos en la observación del concejal de la comuna de Puerto Montt, Fernando España: "Solo se puede apreciar una identidad en la región de Magallanes y parte de Aysén, pero las características existentes en la geografía y composición de sus habitantes son extensivas a la región de Los Lagos" (entrevista con Fernando España, 6 de septiembre de 2014).

Si bien muchos de los encuestados se refirieron, principalmente, a características propias de sus respectivas regiones que luego dio a un análisis general, podemos encontrar dentro de la siguiente opinión una que engloba un sentir que se ha ido posicionando con fuerza dentro de los habitantes de la Patagonia chilena. A juicio del consejero regional de Aysén, Julio Rossel,

no debiera existir una sola identidad en vista de que son muchos territorios y tipos de cultura en función, principalmente, de sus recursos y geografía. Pensar en una sola identidad termina excluyendo a otras de relevancia. En este caso, hablamos de una supraidentidad, donde el componente común es el aislamiento, pero visto desde un punto de vista positivo. (entrevista con Julio Rossel, 6 de septiembre de 2014)

En lo que hace referencia a la pregunta "¿Considera que debiesen de fusionarse las regiones que comprenden la Patagonia chilena?”, llama la atención la opinión dividida que se presenta en el grupo "sociedad civil" con respecto a un eventual proceso de fusión de las regiones en un proyecto macroterritorial con pretensiones alternativas o protonacionalista. En cambio, las autoridades son mayoritariamente contrarias a dicha posibilidad, probablemente por la obvia razón de perder poder al disminuir los espacios de representación (tabla 3).

Tabla 3. ¿Considera que debieran fusionarse las regiones que comprenden la Patagonia chilena? (por grupos de encuestados)

\begin{tabular}{|l|l|l|l|l|}
\hline \multicolumn{2}{|c|}{ Grupos } & \multicolumn{1}{c|}{ No } & \multicolumn{1}{c|}{ Sí } & \multicolumn{1}{c|}{ Total } \\
\hline \multirow{4}{*}{ Autoridades políticas } & Recuento & 20 & 4 & 24 \\
\cline { 2 - 5 } & \% dentro de grupos & 83.3 & 16.7 & 100 \\
\cline { 2 - 5 } & \% del total & 19.4 & 30.9 & 23.3 \\
\hline \multirow{3}{*}{ Sociedad civil } & Recuento & 12 & 11 & 23 \\
\cline { 2 - 5 } & \% dentro de grupos & 52.2 & 47.8 & 100 \\
\cline { 2 - 5 } & \% del total & 11.7 & 10.7 & 22.3 \\
\hline \multirow{5}{*}{ Gobernadores } & Recuento & 2 & 1 & 3 \\
\cline { 2 - 5 } & \% dentro de grupos & 66.7 & 33.3 & 100 \\
\cline { 2 - 5 } & \% del total & 1.9 & 1.0 & 20.9 \\
\hline
\end{tabular}




\begin{tabular}{|c|c|c|c|c|}
\hline \multicolumn{2}{|c|}{ Grupos } & \multirow[t]{2}{*}{ No } & Sí & Total \\
\hline \multirow[t]{3}{*}{ Políticos regionales } & Recuento & & 3 & 7 \\
\hline & $\%$ dentro de grupos & 57.1 & 420.9 & 100 \\
\hline & $\%$ del total & 30.9 & 20.9 & 6.8 \\
\hline \multirow[t]{3}{*}{ Académicos } & Recuento & 8 & 3 & 11 \\
\hline & $\%$ dentro de grupos & 72.7 & 27.3 & 100 \\
\hline & $\%$ del total & 7.8 & 20.9 & 10.7 \\
\hline \multirow[t]{3}{*}{ Municipios Aysén } & Recuento & 9 & 4 & 13 \\
\hline & $\%$ dentro de grupos & 69.2 & 30.8 & 100 \\
\hline & $\%$ del total & 8.7 & 30.9 & 12.6 \\
\hline \multirow[t]{3}{*}{ Municipios Los Lagos } & Recuento & 6 & 5 & 11 \\
\hline & $\%$ dentro de grupos & 54.5 & 45.5 & 100 \\
\hline & $\%$ del total & 5.8 & 40.9 & 10.7 \\
\hline \multirow[t]{3}{*}{ Municipios Magallanes } & Recuento & 8 & 3 & 11 \\
\hline & $\%$ dentro de grupos & 72.7 & 27.3 & 100 \\
\hline & $\%$ del total & 7.8 & 20.9 & 10.7 \\
\hline \multirow{2}{*}{$\begin{array}{l}\text { Total } \\
\% \text { del total }\end{array}$} & Recuento & 69 & 34 & 103 \\
\hline & 67 & 33 & 100 & \\
\hline
\end{tabular}

Fuente: Elaboración propia según Soto (2014).

Por tanto, podemos apreciar en esta tabla una tendencia marcada hacia la negativa de esta respuesta con un total de consultados expresados en $67 \%$ frente a $33 \%$ que considera una respuesta positiva.

Dentro de las argumentaciones que más se repitieron en respuesta a la "no fusión", se presentaron principalmente las de carácter de extensión del territorio, lo cual haría difícil una posible gobernabilidad. Esta apreciación se complementa con la siguiente respuesta representada por Heinrich von Baer, presidente de la Fundación Chile Descentralizado, quien expresa que "son tres territorios demasiados extensos, cuya sede del Gobierno regional quedaría demasiado distante de la gran mayoría de los gobernados" (entrevista con Henrich von Baer, 6 de septiembre de 2014).

Por otra parte, se expresaron argumentos en contra de la fusión de los territorios, ya que se perderían tanto las economías de menor escala como las identidades locales. Dentro de las razones más marcadas, se podrían resumir en la siguiente frase expresada por el exdiputado Pablo Galilea: "Están bien las delimitaciones de las regiones hoy día. Sí debiesen haber trabajos estratégicos en conjunto, mejorar la conectividad marítima y terrestre... sin la necesidad de fusión” (entrevista con Pablo Galilea, 6 de septiembre de 2014).

En cuanto a la respuesta positiva a la pregunta, se puede apreciar, como ya se estableció, que donde hubo mayor tendencia hacia la paridad fue en los grupos Sociedad civil, Municipalidad de Los Lagos y Políticos regionales. En un análisis más detallado, en Sociedad civil la tendencia positiva a esta respuesta se encuentra, principalmente, en las organizaciones sociales y sindicales que la componen. 
En lo que sí hubo acuerdo fue en empoderar las regiones, provincias y comunas contrarrestando la hegemonía del Gobierno central. Mientras que en las propuestas intermedias se destacó la de generar una confederación entre los territorios que comprenden la Patagonia.

\section{Conclusiones}

$\mathrm{Al}$ decir de Robertson (2003), la Patagonia es una región "glocalizada"; está en proceso de constitución de una identidad diferenciada que se ha consolidado con la emergencia de conflictos y actores que reivindican un modelo de desarrollo que tiene nexos con el proceso de globalización que afecta a la región en una doble perspectiva, ecologista y productora de energía, y que la ha integrado a los circuitos de conflictos globales. La Patagonia es una marca en expansión y una delimitación creciente.

El "doble" proceso de debilitamiento del Estado centralista (por "arriba", por efecto de la globalización; por “abajo”, por efecto del proceso de descentralización), respaldado en procesos de fortalecimiento de las autonomías regionales (como se puede observar en la Europa de las regiones), refuerza las demandas por autonomía territorial, especialmente cuando la relación de la Patagonia chilena con los poderes centrales da cuenta de una experiencia de abandono que contrasta con la de las provincias argentinas que tienen condiciones espaciales que se han traducido en más crecimiento.

Esta identidad en construcción se despliega tanto en Chile como en la Patagonia argentina, tal como lo evidencian tanto las iniciativas de cooperación que, en el ámbito fundamentalmente subnacional, se han gestado entre diversos municipios de la macrorregión (los intercambios entre municipios se dan en todo el sur de ambos países, pero son más activos en atención de salud, precisamente, en la región de Aysén, sus vecinos de río Negro y río Chubut) como la identificación de rasgos culturales comunes. Como toda identidad, se construye a partir de un doble movimiento de afirmación de lo propio y de rechazo a lo "foráneo", de componentes nuevos y heredados y trasvasijados, pero que adquieren un significado nuevo en un escenario de empoderamiento territorial fortalecido al alero de los conflictos territoriales de Magallanes y Aysén, aunque con diferentes orientaciones.

Son estas diferentes orientaciones las que hacen de este un proceso conflictivo en su doble dimensión de construcción de identidad y de delimitación territorial. A menos que asumamos una definición esencialista de la identidad que la vincule a ciertas características culturales, consideramos que tiene más relevancia la delimitación territorial en cuanto esta acotará el "quiénes" constituyen el sujeto que definirá las opciones políticas. Dicho desde otra perspectiva, el proyecto autonomista se juega más en la eventual aplicación del principio de autodeterminación de los pueblos que en el obsoleto principio de las nacionalidades.

Respecto de las delimitaciones en disputa, es claramente mayoritaria aquella posición que sostiene que la Patagonia chilena comprende las dos regiones más australes, 
además de la provincia de Palena, perteneciente a la región de Los Lagos. También es mayoritario el respaldo a un trabajo más coordinado con la Patagonia argentina, tanto en el contexto local como provincial-regional y nacional.

Otro ámbito donde se observa un consenso bastante amplio es en lo relativo a la importancia de impulsar un nuevo modelo de desarrollo que releve la protección del medio ambiente y la identidad cultural y deje de lado la matriz extractivista e industrialista. Tras esta disputa, subyace una demanda más sustantiva: que el consenso colectivo construido en torno a esta identidad colectiva (en proceso de consolidación), y que maduró en torno a un conjunto de conflictos territoriales, permita orientar el debate sobre el modelo de desarrollo e influir en el proceso de toma de decisiones políticas, de tal manera que refuerce el alineamiento entre esa identidad, el proyecto colectivo y las decisiones del sistema político.

Donde hay menos acuerdo es respecto de los alcances del proyecto autonomista. La mayor debilidad de este acuerdo gira en torno a dos dimensiones: por un lado, una de carácter territorial que reconoce un mayor apoyo en la sociedad aysenina (en contraste con Magallanes); por otro, una que identifica más apoyo a esta iniciativa en los actores sociales en comparación con los actores gubernamentales.

Aysén como región pequeña en población y poderío económico, además de menor empatía con la acción del Gobierno central chileno, es más proclive tanto a modelos alternativos de desarrollo como a una mayor fusión en la identidad o comunidad inventada de Patagonia.

\section{Referencias}

Anderson, B. (1993). Comunidades imaginadas: reflexiones sobre el origen y la difusión del nacionalismo. México: Fondo de Cultura Económica.

Arocena, J. (1995). El desarrollo local: un desafío contemporáneo. Montevideo: Taurus.

Arocena, J. (2002). El desarrollo local: un desafío contemporáneo (2. ${ }^{a}$ ed.). Montevideo: Taurus.

Banco Mundial (BM) (1997). El estado en un mundo en transformación. Washintong, D. C.: BM.

Bayer, O. (2009). La Patagonia rebelde. Tafalla, España: Txalaparta.

Beck, U. y Grande, E. (2006). La Europa cosmopolita. Barcelona: Paidós.

Billig, M. (2006). Nacionalisme banal. Catarroja, València: Universitat de València.

Bridges, E. L. (1952). El último confín de la tierra. Buenos Aires: Emecé.

Bucciarelli, M. A. (2009). La Patagonia argentina como territorio nacional: perspectivas de análisis. Ponencia presentada en VIII Congreso de Historia Social y Política de la Patagonia Argentino Chilena, Chubut, Universidad Nacional del Comahue. 
Bustos, B. (2012). Brote del virus ISA: crisis ambiental y capacidad de la institucionalidad ambiental para manejar el conflicto. EURE, 38(115), 219-245.

Daher, A. (2003). regiones-commodities: crisis y contagio en Chile. EURE, 29(86), 89-108.

De Agostini, A. (1956). Treinta años en Tierra del Fuego. Buenos Aires: Preuser.

Emperaire, J. (2002). Los nómades del mar. Santiago de Chile: LOM.

Ffrench-Davis, R. (2005). Reformas para América Latina. Santiago de Chile: Siglo XXI.

Garretón, M. A. (2000). La sociedad en qué vivi(re)mos. Santiago: LOM.

Gellner, E. (1988). Naciones y nacionalismo. Madrid: Alianza Editorial.

Giménez, G. (2005). Territorio e identidad: breve introducción a la geografía cultural. Trayectorias, 7(17), 8-24.

Güell, P. (1996). Historia cultural del programa de identidad. Persona y Sociedad, 19(1), 9-28.

Harvey, D. (2004). El “nuevo" imperialismo: acumulación por desposesión. Madrid: Socialist Register.

Hroch, M. (2001). La naturalesa de la nació. Catarroja, València: Universitat de València.

Lacroix, F. (1841). Historia de la Patagonia, Tierra de Fuego e Islas Malvinas. Barcelona: Imprenta del Liberal Barcelonés.

Ladino Tapia, M. y González Gil, A. (eds.) (2014). Regiones fronterizas, migración y los desafíos para los Estados nacionales latinoamericanos. Santiago de Chile: RIL editores.

Laming, A. (1957). En la Patagonia, confin del mundo. Santiago de Chile: Editorial del Pacífico.

Lander, E. (2014). El neoextractivismo como modelo de desarrollo en América Latina y sus contradicciones. Berlín: Fundación Heinrich Boll.

Larraín, J. (2001). Identidad chilena. Santiago de Chile: LOM.

Larraín, J. (2005). ¿América Latina moderna? Globalización e identidad. Santiago de Chile: LOM.

Marimán, J.A. (2012). Autodeterminación: ideas políticas mapuhe en el albor del siglo $X X I$. Santiago de Chile: LOM.

Martinic, M. (1977). Historia del estrecho de Magallanes. Santiago de Chile: Andrés Bello. 
Núñez, P. G., Matossian, B. y Vejsbjerg, L. (2012). Patagonia, de margen exótico a periferia turística: una mirada sobre un área natural protegida de frontera. Pasos: Revista de Turismo y Patrimonio Cultural, 10(1), 47-59.

Muñoz G., O. (ed.) (1992). Después de las privatizaciones: hacia el Estado regulador. Santiago de Chile: Corporación de Investigaciones Económicas para América Latina.

Naím, M. (2013). El fin del poder: empresas que se hunden, militares derrotados, papas que renuncian y gobiernos impotentes: cómo el poder ya no es lo que era. México: Debate.

Navarro Floria, P. (2005). La conquista de la memoria: la historiografía sobre la frontera sur argentina durante el siglo XIX. Universum, 2O(1), 88-111.

Núñez, A., Aliste, E. y Bello, Á. (2014). El discurso del desarrollo en Patagonia-Aysén: la conservación y la protección de la naturaleza como dispositivos de una renovada colonización. Chile, siglos XX-XXI. Scripta Nova: Revista Electrónica de Geografía y Ciencias Sociales, 18.

Núñez Seixas, X. M. (1999). Los nacionalismos en la España contemporánea (siglos $X I X$ y XX). Barcelona: Hipòtesi.

Oszlak, O. (comp.) (1997). Estado y sociedad: las nuevas reglas del juego. Buenos Aires: Universidad de Buenos Aires.

Pacheco Habert, G. S., Vera Garnica, J. y Castaing von der Hundt, J. C. (2015). La gestión de destinos en la región de Los Lagos-Patagonia chilena: ċuna disputa entre asociatividad y competitividad? RIAT: Revista Interamericana de Medioambiente y Turismo, 11(2), 148-162.

Paredes, M. A., Montecino, V., Anic, V., Egaña, M. y Guzmán, L. (2014). Diatoms and dinoflagellates macroscopic regularities shaped by intrinsic physical forcing variability in Patagonian and Fuegian fjords and channels (48-56 S). Progress in Oceanography, 129, 85-97.

Penaglia Vásquez, F. E. y Valenzuela van Treek, E. (2014). Rebeldía en Calama: desafío al orden centralista chileno en un contexto de boom minero. Revista Mexicana de Ciencias Políticas y Sociales, 59(222), 161-185.

Portillo Riascos, L. H. (2014). Extractivismo clásico y neoextractivismo: ¿dos tipos de extractivismos diferentes? Tendencias, 15(2), 11-29.

Ramos Posek, V. (2014). La responsabilidad social de megaempresas y Estado en el uso y administración de los bienes comunes: los proyectos societales y el nuevo colonialismo en América Latina. El caso de la intervención de la mega-industria 
del salmón en los bordes costeros del archipiélago de Chiloé, Chile. 1983-2013 (Tesis de doctorado, Universidad ARCIS, Santiago, Chile).

Robertson, R. (2003). Glocalización: tiempo-espacio y homogenidad-heterogeneidad. En J. C. Monedero Fernández-Gala (coord.), Cansancio del Leviatán: problemas políticos de la mundialización (pp. 261-284). Madrid: Trotta.

Rojas Böttner, A. (2013). Expansión centralista y exclusión regional. Santiago de Chile: Instituto Chileno de Estudios Municipales.

Rojas Böttner, A. (2013). Movimientos regionalistas, expansión estatal y sistema de partidos: los casos de la revolución constituyente de Atacama (1859) y el partido regionalista de Magallanes (1932-1952) (Tesis de maestría, Universidad Alberto Hurtado, Santiago de Chile).

Saz Campos, I. y Romeo Mateo, M. C. (2004). Construir Espanya al segle XIX. Afers, 19(48), 261-263.

Soto, G. (2014). Delimitación de la Patagonia chilena: imaginarios, ecología y lucha social (Tesis de grado, Universidad Alberto Hurtado Universidad, Santiago de Chile).

Smith, A. D. (1991). The ethnic origins of nations. Basil: Blackwell.

Tapia Ladino, M. y González Gil, A. (2014). Regiones froterizas: migración y los desafíos para los estados latinoamericanos. Santiago de Chile: RIL editorres.

Tomassini, L. (1995). La reforma del Estado y las políticas públicas. Santiago de Chile: Universidad de Chile.

Valenzuela, E. (2015). Territorios rebeldes. Santiago de Chile: Universidad Alberto Hurtado Universidad.

Waidhofer, L. (2011). La Patagonia desconocida. Western Eye Press. 